\title{
Spin observables in three-body direct nuclear reactions
}

\author{
A. Deltuva \\ Centro de Física Nuclear da Universidade de Lisboa, P-1649-003 Lisboa, Portugal
}

\begin{abstract}
Direct nuclear reactions $\vec{d}+A$ and $\vec{p}+(A n)$ are described in the framework of three-body Faddeev-type equations. Differential cross section and analyzing powers are calculated using several optical potential models and compared with the experimental data. Quite satisfactory agreement is found except for few systematic discrepancies.
\end{abstract}

Key words: three-body scattering, Faddeev equations, analyzing power PACS: 24.10.-i, 21.45.+v, 24.70.+s, 25.55.Ci

\section{Introduction}

Direct nuclear reactions, dominated by three-body degrees of freedom, provide an important test for the models of nuclear dynamics. In the past, the reactions like the deuteron $(d)$ scattering from a stable nucleus $(A)$ were described using the approximate continuum discretized coupled channels (CDCC) method [1]. With few exceptions the spin degrees of freedom have been usually neglected. In such a case one can only calculate the unpolarized cross sections which are mostly sensitive to the central part of the nucleon-nucleus optical potential. However, there exist experimental data for polarization observables that depend as well on the spin-orbit part of the optical potential. The aim of the present work is to study the spin observables in $\vec{d}+A$ and $\vec{p}+(A n)$ reactions in the framework of exact three-body Faddeev/Alt, Grassberger, and Sandhas (AGS) equations [2, 3] such that all discrepancies with the experimental data can be attributed solely to the shortcomings of the used optical potential models. A part of the reactions to be considered here have already been studied by the CDCC method [4] .

Email address: deltuva@cii.fc.ul.pt (A. Deltuva) 
Thus, the comparison with the results of Ref. [4] may allow us to draw some conclusions on the reliability of CDCC for calculating spin observables, in addition to the cross section benchmark [5].

The theoretical framework is shortly recalled in Sec. 2, the results are presented in Sec. 3, and the summary is given in Sec. 4 .

\section{Faddeev/AGS equations and dynamic input}

We describe $\vec{d}+A$ and $\vec{p}+(A n)$ type reactions using three-body $(p, n, A)$ model. An exact treatment of the quantum three-body scattering problem is provided by both Faddeev [2] and AGS equations [3] that are equivalent to the Schrödinger equation but are more suitable for the numerical solution due to the connectedness of the kernel. The Faddeev equations are formulated for the components of the wave function while the AGS equations,

$$
U_{\beta \alpha}=\bar{\delta}_{\beta \alpha} G_{0}^{-1}+\sum_{\sigma=1}^{3} \bar{\delta}_{\beta \sigma} T_{\sigma} G_{0} U_{\sigma \alpha}
$$

are a system of coupled integral equations for the transition operators $U_{\beta \alpha}$ whose on-shell matrix elements $\left\langle\psi_{\beta}\left|U_{\beta \alpha}\right| \psi_{\alpha}\right\rangle$ are scattering amplitudes and therefore lead directly to the observables. In Eq. (1) $\bar{\delta}_{\beta \alpha}=1-\delta_{\beta \alpha}, G_{0}=$ $\left(E+i 0-H_{0}\right)^{-1}$ is the free resolvent, and $T_{\sigma}=v_{\sigma}+v_{\sigma} G_{0} T_{\sigma}$ is the two-particle transition matrix, $E$ being the available three-particle energy in the center of mass (c.m.) system, $H_{0}$ the free Hamiltonian, and $v_{\sigma}$ the potential for the pair $\sigma$ in odd-man-out notation. The channel states $\left|\psi_{\sigma}\right\rangle$ for $\sigma=1,2,3$ are the eigenstates of the corresponding channel Hamiltonian $H_{\sigma}=H_{0}+v_{\sigma}$ with the energy eigenvalue $E$; thus, $\left|\psi_{\sigma}\right\rangle$ is a product of the bound state wave function for pair $\sigma$ and a plane wave with fixed on-shell momentum corresponding to the relative motion of particle $\sigma$ and pair $\sigma$ in the initial or final state. Observables of elastic scattering are calculated from the matrix elements with $\beta=\alpha$ while $\beta \neq \alpha$ corresponds to transfer reactions.

We solve the AGS equations using momentum-space partial-wave basis where they become a system of integral equations with two continuous variables, the values of Jacobi momenta. The employed numerical techniques are described in great detail in Refs. [6, 7, 8].

The AGS equations are applicable only to short-range potentials $v_{\sigma}$. Nevertheless, the long-range Coulomb force between charged particles can be included in this framework using the method of screening and renormalization 
[9, 10, 11] which enables to calculate the Coulomb-distorted short-range part of the transition amplitude by solving the AGS equations with nuclear plus screened Coulomb potential; the convergence of the results with the screening radius has to be established. The method has been successfully applied to proton-deuteron [8, 12] and $\alpha$-deuteron [13] elastic scattering and breakup, and to three-body nuclear reactions involving deuterons or one-neutron halo nuclei [5].

The dynamic input to the AGS equations are the potentials $v_{\sigma}$ for the three pairs of particles. As the $n p$ interaction we take realistic CD Bonn potential [14], in contrast to the usual CDCC calculations where a simple Gaussian $n p$ potential is used. For the nucleon-nucleus $(N A)$ interaction, in order to study the model dependence, we use several different optical potentials, namely, those by Watson et al. [15], Menet et al. [16], Becchetti and Greenlees [17], and Koning and Delaroche [18]; the corresponding predictions in the following will be abbreviated by $\mathrm{W}, \mathrm{M}, \mathrm{BG}$, and $\mathrm{KD}$, respectively. Each of these potentials is fitted to the $N A$ data in a limited mass and energy range; those limitations are respected in the present calculations. The energy-dependent parameters of the potentials are taken at a half deuteron lab energy in the $\vec{d}+A$ reactions and at the proton lab energy for $p A$ in the $\vec{p}+(A n)$ reactions. In the latter case the $n A$ potential is real and supports a number of bound states corresponding to the ground and excited single-particle states of the $(A n)$ nucleus while all Pauli forbidden states are removed; the potential parameters and the resulting binding energies are given in Ref. [19] for ${ }^{13} \mathrm{C}$ and ${ }^{17} \mathrm{O}$ nuclei. The interaction within $n p, n A$, and $p A$ pairs is included in partial waves with pair orbital angular momentum $L \leq 3,10$, and 20, respectively, and the total angular momentum is $J \leq 45$; depending on the reaction some of these quantum numbers cutoffs can be safely chosen significantly lower, leading, nevertheless, to well converged results. The $p A$ channel is more demanding than the $n A$ channel due to the screened Coulomb force, where the screening radius $R \approx 8$ to $10 \mathrm{fm}$ for the short-range part of the scattering amplitude is sufficient for the convergence.

\section{Results}

The experimental data for the spin observables are scarcer than for the spin-averaged cross sections. Nevertheless, a complete set of deuteron analyzing powers over a wide mass and angular range is presented in Ref. [20] for $E_{d}=56 \mathrm{MeV}$; other measurements exist as well but cover only rather 

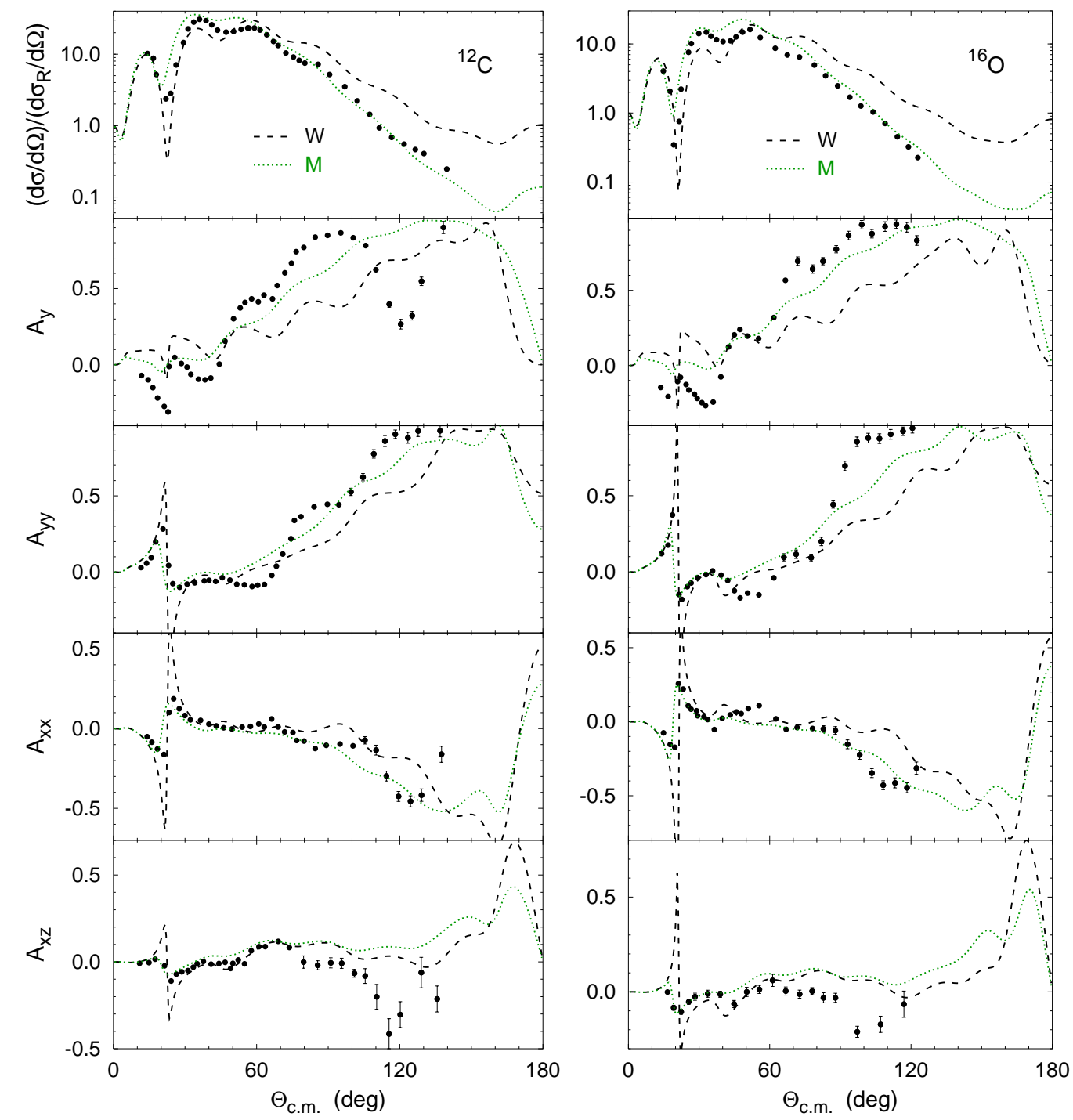

Figure 1: Differential cross section divided by Rutherford cross section and deuteron analyzing powers for deuteron elastic scattering from ${ }^{12} \mathrm{C}$ and ${ }^{16} \mathrm{O}$ nuclei at $E_{d}=56 \mathrm{MeV}$ as functions of the c.m. scattering angle. Results for $N A$ potentials $\mathrm{W}$ and $\mathrm{M}$ are given by dashed and dotted curves, respectively. The experimental data are from Ref. 20].

narrow angular or mass range. We therefore concentrate first on $56 \mathrm{MeV}$ polarized deuteron scattering from ${ }^{12} \mathrm{C},{ }^{16} \mathrm{O},{ }^{28} \mathrm{Si},{ }^{40} \mathrm{Ca}$, and ${ }^{58} \mathrm{Ni}$ nuclei. The results are presented in Figs. 1 and 2 , 

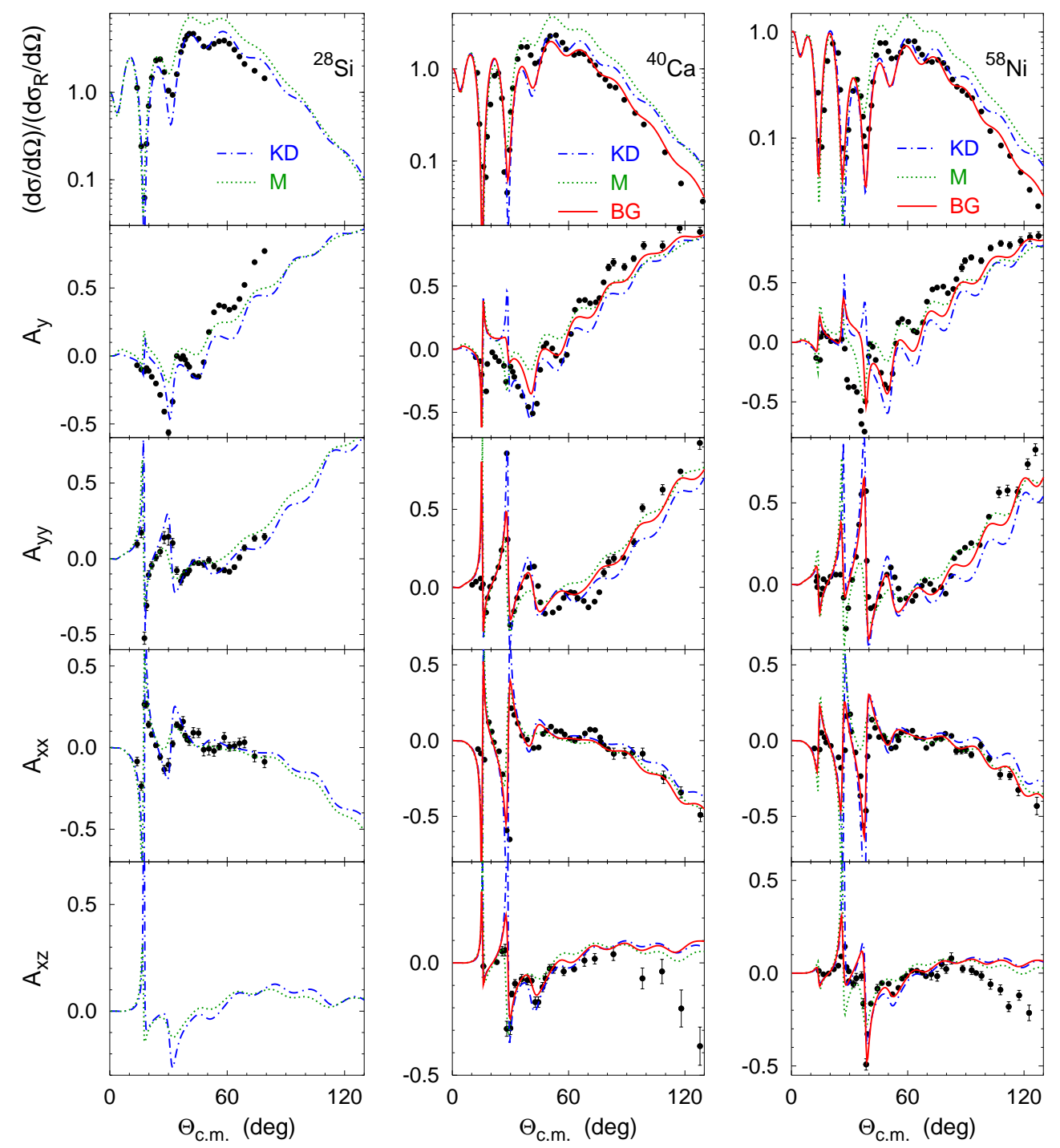

Figure 2: Differential cross section divided by Rutherford cross section and deuteron analyzing powers for deuteron elastic scattering from ${ }^{28} \mathrm{Si},{ }^{40} \mathrm{Ca}$, and ${ }^{58} \mathrm{Ni}$ nuclei at $E_{d}=56$ $\mathrm{MeV}$. Results obtained with $N A$ potentials $\mathrm{KD}, \mathrm{M}$, and $\mathrm{BG}$ are given by dashed-dotted, dotted, and solid curves, respectively. The experimental data are from Ref. [21] for ${ }^{28} \mathrm{Si}$ and from Ref. 20] for other nuclei. 
As shown in Fig. 1, in the case of ${ }^{12} \mathrm{C}$ and ${ }^{16} \mathrm{O}$ nuclei the predictions using the potential $\mathrm{M}$ describe the experimental data better than those of $\mathrm{W}$, especially at larger scattering angles. There are significant differences also around $\Theta_{\text {c.m. }}=22$ deg where $\mathrm{W}$ predicts much deeper minimum in the cross section and thereby too sharp peaks in the analyzing powers; in contrast, the corresponding peaks obtained with $\mathrm{M}$ for ${ }^{12} \mathrm{C}$ are not sharp enough. Both potentials fail to reproduce the vector analyzing power $A_{y}$ data at small angles $\Theta_{c . m .}<40 \mathrm{deg}$; the reason for this failure, at least to some extent, may be the inability to describe $A_{y}$ at small angles in $\vec{p}+A$ elastic scattering as can be seen in Ref. [15]. The $A_{y}$ and $A_{y y}$ data at intermediate angles are slightly underpredicted, and, in addition, $A_{y}$ for ${ }^{12} \mathrm{C}$ shows a clear minimum around $\Theta_{c . m .}=120$ deg that is not present in the theoretical results. Furthermore, calculated $A_{x z}$ for both ${ }^{12} \mathrm{C}$ and ${ }^{16} \mathrm{O}$ nuclei at larger angles deviates quite significantly from the data. Otherwise the qualitative description of the data by the potential M is quite satisfactory. The comparison with the $\vec{d}+{ }^{12} \mathrm{C}$ data from Ref. [22] at $E_{d}=35$ to $70 \mathrm{MeV}$ that are limited to narrow angular range from 35 to 80 deg (not shown here) brings essentially the same conclusions.

However, the potential $\mathrm{M}$ fails in the case of heavier nuclei ${ }^{28} \mathrm{Si},{ }^{40} \mathrm{Ca}$, and ${ }^{58} \mathrm{Ni}$ as shown in Fig. 2. It clearly overpredicts the differential cross section at $\Theta_{\text {c.m. }}>40 \mathrm{deg}$ while the analyzing power data are reproduced with similar quality as by other potentials. Quite surprisingly, at larger angles $\Theta_{\text {c.m. }}>60$ deg the old global potential BG describes deuteron scattering from ${ }^{40} \mathrm{Ca}$ and ${ }^{58} \mathrm{Ni}$ nuclei better than the new and precise $\mathrm{KD}$ potential which has many more parameters fitted individually to the $n A$ and $p A$ data of the considered nuclei. At smaller angles all potentials account for the data with comparable quality except for $A_{y}$ where KD shows an additional sharp maximum instead of a minimum. The most serious discrepancies between theory and data take place in small-angle $A_{y}$ and in large-angle $A_{x z}$. While the latter one is similar as in the case of ${ }^{12} \mathrm{C}$ and ${ }^{16} \mathrm{O}$ nuclei, the former one is somehow different: the data are reproduced at very small angles, but then there is a narrow angular interval around $\Theta_{\text {c.m. }}=30 \mathrm{deg}$ where $A_{y}$ is strongly overpredicted. Furthermore, in contrast to ${ }^{12} \mathrm{C}$ and ${ }^{16} \mathrm{O}, A_{y}$ in proton scattering from heavier nuclei is well described by the employed potentials. Thus, in this case the $A_{y}$ discrepancy even partially cannot be explained by a poor $N A$ data fit and must be due to another, presently unknown, reason. In addition, $A_{y}$ and $A_{y y}$ at larger angles are slightly underpredicted but the oscillating behaviour of the observables is reproduced rather well. Otherwise the qualitative description of the data, especially by the potential BG, is 

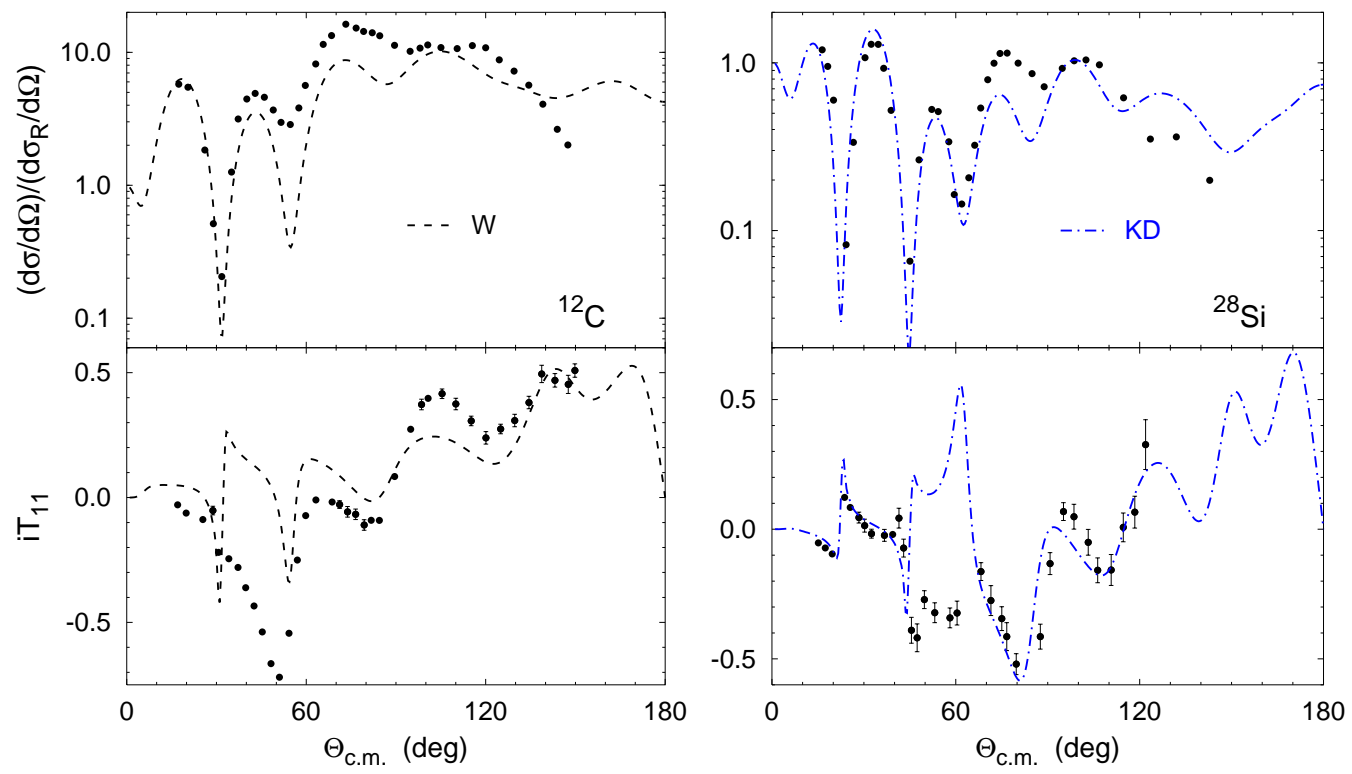

Figure 3: Differential cross section divided by Rutherford cross section and deuteron vector analyzing power $i T_{11}$ for deuteron elastic scattering from ${ }^{12} \mathrm{C}$ and ${ }^{28} \mathrm{Si}$ nuclei at $E_{d}=29.5$ $\mathrm{MeV}$ calculated using the potentials $\mathrm{W}$ and $\mathrm{KD}$, respectively. The experimental data are from Ref. [23].

quite satisfactory.

Even larger discrepancy in the vector analyzing power $A_{y}$ or, equivalently, $i T_{11}=A_{y} \sqrt{3} / 2$, is present at lower energies as shown in Fig. 3 . for the elastic deuteron scattering from ${ }^{12} \mathrm{C}$ and ${ }^{28} \mathrm{Si}$ nuclei at $E_{d}=29.5 \mathrm{MeV}$. Compared to Figs. 1 1 and 2, the discrepancy takes place at larger scattering angles, 30 to $55 \mathrm{deg}$ and 45 to $70 \mathrm{deg}$ for ${ }^{12} \mathrm{C}$ and ${ }^{28} \mathrm{Si}$, respectively. Outside those regions the calculations account for the data quite satisfactorily, at least in the case of ${ }^{28} \mathrm{Si}$. The agreement for the differential cross section is rather similar to the one seen at $E_{d}=56 \mathrm{MeV}$.

Deuteron scattering from ${ }^{16} \mathrm{O},{ }^{40} \mathrm{Ca}$, and ${ }^{58} \mathrm{Ni}$ nuclei at $E_{d}=56 \mathrm{MeV}$ was calculated in Ref. [4] in the CDCC framework using M and BG optical potentials. However, the $n p$ interaction in Ref. [4] and in the present work is not exactly the same. In Ref. [4] the deuteron bound state was calculated with a realistic $n p$ potential but the $n p$ continuum was described by simple Gaussian potential acting in the triplet S and D waves only. Such a mixed potential cannot be included in consistent Faddeev-type calculations such 
as of the present work. Nevertheless, since the considered observables are rather insensitive to the $n p$ potential, a comparison of the present results and those of Ref. [4] is meaningful. The agreement is good, there are only rather small differences, e.g., our $A_{y}$ values for $\Theta_{c . m .} \geq 60 \mathrm{deg}$ are higher by about $0.05-0.10$ coming closer to the data. Thus, in addition to the cross section benchmark [5] one can conclude that CDCC is quite reliable also in calculating the spin observables in deuteron-nucleus elastic scattering.

The $\vec{p}+(A n)$ elastic scattering is a less interesting case since the differential cross section and the proton analyzing power are quite strongly correlated with the corresponding observables in $\vec{p}+A$ elastic scattering to which the $p A$ potential is fitted. The data for other observables like $(A n)$ analyzing power and spin correlation coefficients does not exist. Furthermore, the only available polarization data refer to proton- ${ }^{13} \mathrm{C}$ scattering. Examples at proton lab energy $E_{p}=17.5$ and $35 \mathrm{MeV}$ are shown in Fig. [4 together with the differential cross section. The calculations describe the experimental data with the same quality as for the corresponding proton $-{ }^{12} \mathrm{C}$ observables and even show similar deviations [15], e.g., underestimation of the large angle cross section at $17.5 \mathrm{MeV}$ and overestimation of the small angle $A_{y}$. However, in contrast to the deuteron- ${ }^{12} \mathrm{C}$ scattering, it seems that the potential $\mathrm{W}$ describes the data slightly better than $\mathrm{M}$, except for forward angles.

In the Faddeev/AGS framework the elastic scattering and the transfer reaction $\vec{p}+(A n) \rightarrow d+A$ are calculated simultaneously. Unfortunately, the data only exists for $\vec{p}+{ }^{13} \mathrm{C} \rightarrow d+{ }^{12} \mathrm{C}$ transfer at $E_{p}=65 \mathrm{MeV}$. Although this is slightly above the upper validity limit of the employed optical potentials $\mathrm{W}$ and $\mathrm{M}$ and both of them do not reproduce accurately the $p A$ data at this energy, we show the results in Fig. 5. The description of the data is poor for both differential cross section and proton analyzing power, and is slightly worse when using the potential M. Note that a similar failure in the transfer cross section is observed in the $d+{ }^{16} \mathrm{O} \rightarrow p+{ }^{17} \mathrm{O}$ reaction at $E_{d}=63.2 \mathrm{MeV}$ [19].

\section{Summary}

We performed Faddeev-type calculations of three-body direct nuclear reactions in $(p, n, A)$ model. The framework is the integral momentum-space AGS equations; the Coulomb interaction between the charged particles is included using the method of screening and renormalization; well converged results are obtained. Thus, all discrepancies with the experimental data can 

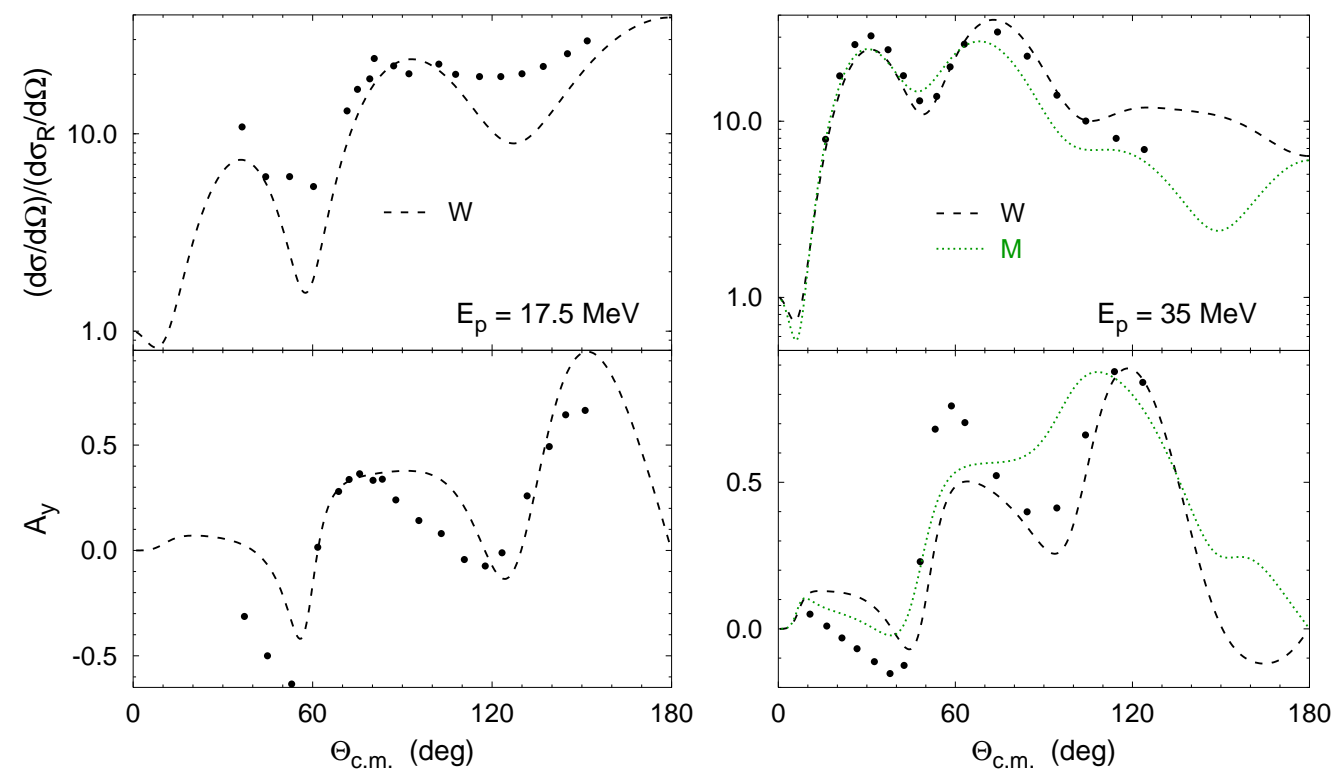

Figure 4: Differential cross section divided by Rutherford cross section and proton analyzing power for proton- ${ }^{13} \mathrm{C}$ elastic scattering at $E_{p}=17.5$ and $35 \mathrm{MeV}$. Curves as in Fig. 1 The experimental data are from Refs. 24] and 25] at 17.5 and $35 \mathrm{MeV}$, respectively.
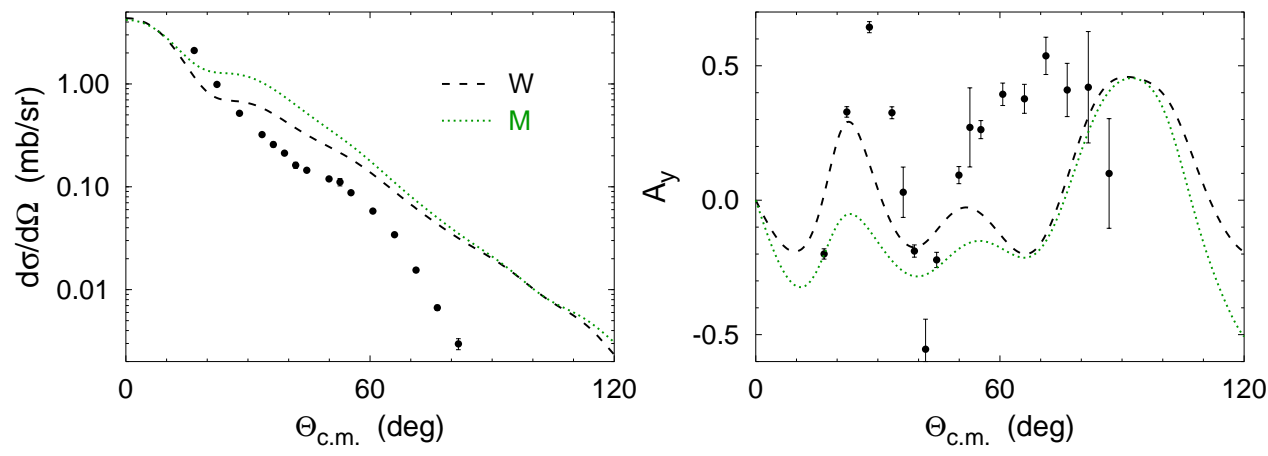

Figure 5: Differential cross section and proton analyzing power for $\vec{p}+{ }^{13} \mathrm{C} \rightarrow d+{ }^{12} \mathrm{C}$ reaction at $E_{p}=65 \mathrm{MeV}$. Curves as in Fig. 11. The experimental data are from Ref. [26]. 
be attributed to the shortcomings of the used optical potentials or even to the inadequacy of the three-body model. A realistic $n p$ potential and several parametrizations of the nucleon-nucleus optical potentials were used, all including spin-orbit interaction. Differential cross section and analyzing powers were calculated for the deuteron elastic scattering from ${ }^{12} \mathrm{C},{ }^{16} \mathrm{O},{ }^{28} \mathrm{Si},{ }^{40} \mathrm{Ca}$, and ${ }^{58} \mathrm{Ni}$ nuclei, and for $\vec{p}+{ }^{13} \mathrm{C}$ elastic scattering and transfer to $d+{ }^{12} \mathrm{C}$. The description of the experimental data is mostly quite satisfactory, at least by some of the optical potential models, and is of similar quality as in the nucleon-nucleus elastic scattering to which the optical potentials are fitted. Systematic discrepancies are found in elastic proton and deuteron vector analyzing power $A_{y}$ at small angles, and in the deuteron tensor analyzing power $A_{x z}$ at large angles. The former one, in the case of light nuclei ${ }^{12} \mathrm{C}$ and ${ }^{16} \mathrm{O}$, may be related to a similar problem in the nucleon-nucleus $A_{y}$. The calculations also fail in accounting for the available $\vec{p}+{ }^{13} \mathrm{C} \rightarrow d+{ }^{12} \mathrm{C}$ transfer data. A satisfactory qualitative agreement with previous CDCC results for $\vec{d}+A$ elastic observables is found indicating the reliability of the CDCC method for this type of reactions.

\section{Acknowledgements}

The author thanks A. C. Fonseca for comments on the manuscript. The work is supported by the Fundação para a Ciência e a Tecnologia (FCT) grant SFRH/BPD/34628/2007.

\section{References}

[1] N. Austern, Y. Iseri, M. Kamimura, M. Kawai, G. Rawitscher, M. Yahiro, Phys. Rep. 154 (1987) 125.

[2] L. D. Faddeev, Zh. Eksp. Theor. Fiz. 39 (1960) 1459 [Sov. Phys. JETP 12 (1961) 1014].

[3] E. O. Alt, P. Grassberger, W. Sandhas, Nucl. Phys. B2 (1967) 167.

[4] Y. Iseri, H. Kameyama, M. Kamimura, M. Yahiro, M. Tanifuji, Nucl. Phys. A 490 (1988) 383.

[5] A. Deltuva, A. M. Moro, E. Cravo, F. M. Nunes, A. C. Fonseca, Phys. Rev. C 76 (2007) 064602. 
[6] K. Chmielewski, A. Deltuva, A. C. Fonseca, S. Nemoto, P. U. Sauer, Phys. Rev. C 67 (2003) 014002.

[7] A. Deltuva, K. Chmielewski, P. U. Sauer, Phys. Rev. C 67 (2003) 034001.

[8] A. Deltuva, A. C. Fonseca, P. U. Sauer, Phys. Rev. C 71 (2005) 054005; A. Deltuva, A. C. Fonseca, P. U. Sauer, Phys. Rev. C 72 (2005) 054004; A. Deltuva, A. C. Fonseca, P. U. Sauer, Phys. Rev. C 73 (2006) 057001.

[9] J. R. Taylor, Nuovo Cimento B23 (1974) 313;

M. D. Semon, J. R. Taylor, Nuovo Cimento A26 (1975) 48.

[10] E. O. Alt, W. Sandhas, Phys. Rev. C 21 (1980) 1733.

[11] A. Deltuva, A. C. Fonseca, P. U. Sauer, Annu. Rev. Nucl. Part. Sci. 58 (2008) 27.

[12] A. Deltuva, A. C. Fonseca, P. U. Sauer, Phys. Rev. Lett. 95 (2005) 092301.

[13] A. Deltuva, Phys. Rev. C 74 (2006) 064001.

[14] R. Machleidt, Phys. Rev. C 63 (2001) 024001.

[15] B. A. Watson, P. P. Singh, R. E. Segel, Phys. Rev. 182 (1969) 978.

[16] J. J. H. Menet, E. E. Gross, J. J. Malanify, A. Zucker, Phys. Rev. C 4 (1971) 1114.

[17] F. D. Becchetti Jr., G. W. Greenlees, Phys. Rev. 182 (1969) 1190.

[18] A. J. Koning, J. P. Delaroche, Nucl. Phys. A 713 (2003) 231.

[19] A. Deltuva, A. C. Fonseca, Phys. Rev. C 79 (2009) 014606.

[20] N. Matsuoka, H. Sakai, T. Saito, K. Hosono, M. Kondo, H. Ito, K. Hatanaka, T. Ichihara, A. Okihana, K. Imai, K. Nisimura, Nucl. Phys. A 455 (1986) 413.

[21] K. Hatanaka, K. Imai, S. Kobayashi, T. Matsusue, M. Nakamura, K. Nisimura, T. Noro, H. Sakamoto, H. Shimizu, J. Shirai, Nucl. Phys. A 340 (1980) 93. 
[22] S. Kato, N. Matsuoka, T. Noro, T. Saito, H. Sakai, M. Nakamura, M. Yosoi, T. Ichihara, K. Hatanaka, H. Ogawa, Nucl. Instr. Meth. A 238 (1985) 453.

[23] G. Perrin, N. V. Sen, J. Arvieux, R. Darves-Blanc, J. Durand, A. Fiore, J. Gondrand, F. Merchez, C. Perrin, Nucl. Phys. A 282 (1977) 221.

[24] H. R. Weller, J. Szücs, P. G. Ikossi, J. A. Kuehner, D. T. Petty, R. G. Seyler, Phys. Rev. C 18 (1978) 1120.

[25] H. Ohnuma, B. A. Brown, D. Dehnhard, K. Furukawa, T. Hasegawa, S. Hayakawa, N. Hoshino, K. Ieki, M. Kabasawa, K. Maeda, K. Miura, K. Muto, T. Nakagawa, K. Nisimura, H. Orihara, T. Suehiro, T. Tohel, M. Yasue, Nucl. Phys. A 456 (1986) 61.

[26] K. Hosono, M. Kondo, T. Saito, N. Matsuoka, S. Nagamachi, T. Noro, H. Shimizu, S. Kato, K. Okada, K. Ogino, Y. Kadota, Nucl. Phys. A 343 (1980) 234. 